ISSN 1112-9867

\title{
EFFECT OF HOST POLYMER BLENDS TO PHOSPHORESCENCE EMISSION
}

\author{
A. N. Alias ${ }^{1, *}$, Z. M. Zabidi ${ }^{1}$, A. M. M. Ali $^{2}$ and M. Z. A. Yahya ${ }^{3}$ \\ ${ }^{1}$ Faculty of Applied Science, UniversitiTeknologi MARA, 35400 Tapah, Perak, Malaysia \\ ${ }^{2}$ Ionic Materials and Devices (iMADE) Research Laboratory, Institute of Sciences, \\ UniversitiTeknologi MARA, 40450 Shah Alam, Selangor, Malaysia \\ ${ }^{3}$ Faculty of Defence Science and Technology, UniversitiPertahananNasional Malaysia, \\ SungaiBesi Camp, 57000 Kuala Lumpur, Malaysia
}

Published online: 10 November 2017

\begin{abstract}
Thin film poly (N-vinylcarbazole) blend with poly(vinylidene fluoride-co-hexafluoropropene) (PVDF-HfP) and polyvinylpyrrolidone (PVP) contain 5\% weight ratio of Europium (III) Tris(dibenzoylmethane) mono(5-amino-1,10-phenanthroline) was prepared by using doctor blade technique on the spectrosil substrate. Each polymer was blended with the same ratio composition. The influences of host polymer composition to the phosphorescence emission were observed under pulsed UV excitation source of Xenon lamp. The result shows that there were changing in the phosphorescence emission and life time with difference host polymer. The explanation of phosphorescence emission has been supported by ZINDO calculation.
\end{abstract}

Keywords: luminescence; phosphorescence; polymer blend.

Author Correspondence, e-mail: ahmadnazib111@perak.uitm.edu.my

doi: http://dx.doi.org/10.4314/jfas.v9i6s.39 


\section{INTRODUCTION}

Increasing pursuit of electronic and opto-electronic polymer-based devices has led researchers to seek new polymeric materials which fulfill specific requirement on electronic application. Polymer light emitting diode (PLED) is a device based on electrically driven emission of light from amorphous or disordered organic polymeric semiconducting materials. Researches on the polymer light emitting diode have rapidly been grown since in [1] had invented the first PLED using poly(phenylenevinylene) (PPV). The reasons to develop polymer-based light emitting diode are due to low cost, high performance and flexibility. Combination of polymer with metal complex materials have widely been used in light emitting diode. These combinations create long life time due to generate of triplet spin which is called as phosphorescence. While, the material shows fast luminescence life time, it is called fluorescence. There are a lot of metal complexes such as platinum (Pt), Iridium (Ir) and rare earth complex have been applied to the polymer for developing phosphorescence polymer light emitting diode (PhPLED) [2-3]. Among of them, Eu(III) complex has been used as phosphorescent material in PLED which shows excellent properties such as high photoluminescent efficiency, high color purity, independency of main emission peak with ligand change and lower cost than other noble metals [4].

For further application phosphorescence PLED (PhPLED), high color stability can be achieved if there is aggregation between host polymer and phosphorescence dye [5]. Low efficient of energy transfer to the phosphorescent complexes is the main issue which hindering for phosphorescence device application [6]. Therefore, understanding of the electrical and optical properties of host polymer is crucial to improve the performance of further phosphorescence PLEDs. For its future use in phosphorescence polymer light emitting diode as well as electrochemical cell, this present work investigates the effect of host polymers which consist of PVK:PVDF-HfP and PVK:PVP polymer blends to the

phosphorescence emission of Europium (III)Tris(dibenzoylmethane) mono(5-amino-1,10-phenanthroline) (EuDBMPhen). The detail investigated of host polymer can be found in the literature [7-11] 


\section{METHODOLOGY}

Two polymer blending systems consist of blended PVK with PVDF-HFP (PVK:PVDF-HFP) and PVK with PVP (PVK:PVP) in the same weight ratio 1:1 were prepared. Meanwhile, pure PVK was also prepared as a control. PVK, PVK:PVDF-HFP and PVK:PVP were dissolved in $10 \mathrm{ml}$ dimethylformamide (DMF) common solvent and stirred for 6 hours at room temperature $\left(30^{\circ} \mathrm{C}\right)$. Then, $5 \%$ weight ratio of Europium (III) Tris(dibenzoylmethane) mono(5-amino-1,10-phenanthroline) was adding into the host polymer. The solution was stirred again for 6 hours at room temperature $\left(30^{\circ} \mathrm{C}\right)$. Then, each solution was coated on a spectrosil substrate using doctor blade technique to form a thin film. The films were then dried in vacuum oven at $50 \mathrm{oC}$ for an hour. Prior to coating, the spectrosil substrate was cleaned with Decon90, rinsed with deionized water and ultrasonicated with acetone. The thickness of the thin films was measured by using reflectometry spectroscopy filmetrics F20 Series. The average thickness of PVK:EuDBMPhen, PVK:PVDF-HFP:EuDBMPhen, PVK:PVP:EuDBMPhen thin films were 410.0, 420.0 and $420.0 \mathrm{~nm}$ respectively. The photoluminescence measurement was conducted using JobinYvon Horiba Spex Fluorolog-3. The samples were placed perpendicular with $450 \mathrm{~W}$ Xenon lamp as excitation source. For phosphorescence analysis, flash xenon lamp with excitation pulsed is $3 \mu \mathrm{s}$.

The electronic properties of polymers and the ligand group EuDBMPhen also has been calculated using semi-empirical calculation. The molecular geometry of carbazole, pyrrolidone, Vinylidene fluoride monomer, dibenzoylmethane (DBM) and mono(5-amino-1,10-phenanthroline) (Phen) was drawn by using worksheet Material Studio. After the desired molecular structure was drawn, geometry optimization has been carrying by using PM3 with single configuration interaction. From the geometry optimization, the electronic calculation and transition energy of arbazole, pyrrolidone, Vinylidene fluoride monomer, dibenzoylmethane (DBM) and mono(5-amino-1,10-phenanthroline) (Phen) was calculated by using Zerner Modification intermediate neglect of differential Overlap (ZINDO) calculation[12].

\section{RESULTS AND DISCUSSION}

The emission spectra of solid state Eu complex (EuDBMPhen) blended with PVK, 
PVK:PVDF-HfP and PVK:PVP at room temperature is shown in Fig. 1. The emission spectra of all samples show the character of Eu complex. For Eu complex blended with PVK the characteristic emission is $575.0 \mathrm{~nm}\left({ }^{5} \mathrm{D}_{0} \rightarrow{ }^{7} \mathrm{~F}_{0}\right), 587.0 \mathrm{~nm}\left({ }^{5} \mathrm{D}_{0} \rightarrow{ }^{7} \mathrm{~F}_{1}\right), 608.5 \mathrm{~nm}\left({ }^{5} \mathrm{D}_{0} \rightarrow{ }^{7} \mathrm{~F}_{2}\right)$ and $649.5 \mathrm{~nm}\left({ }^{5} \mathrm{D}_{0} \rightarrow{ }^{7} \mathrm{~F}_{7}\right)$. While for Eu complex blended with PVK:PVDF-HfP depicts 576.0 $\mathrm{nm}\left({ }^{5} \mathrm{D}_{0} \rightarrow{ }^{7} \mathrm{~F}_{0}\right), 586.5 \mathrm{~nm}\left({ }^{5} \mathrm{D}_{0} \rightarrow{ }^{7} \mathrm{~F}_{1}\right), 608.5 \mathrm{~nm}\left({ }^{5} \mathrm{D}_{0} \rightarrow{ }^{7} \mathrm{~F}_{2}\right)$ and $648.5 \mathrm{~nm}\left({ }^{5} \mathrm{D}_{0} \rightarrow{ }^{7} \mathrm{~F}_{7}\right)$. Finally, for Eu complex blended with PVK:PVP shows $578.5 \mathrm{~nm}\left({ }^{5} \mathrm{D}_{0} \rightarrow{ }^{7} \mathrm{~F}_{0}\right), 586.5 \mathrm{~nm}\left({ }^{5} \mathrm{D}_{0} \rightarrow{ }^{7} \mathrm{~F}_{1}\right)$, $608.5 \mathrm{~nm}\left({ }^{5} \mathrm{D}_{0} \rightarrow^{7} \mathrm{~F}_{2}\right)$ and $648.0 \mathrm{~nm}\left({ }^{5} \mathrm{D}_{0} \rightarrow^{7} \mathrm{~F}_{7}\right)$. The highest emission intensity is located at ${ }^{5} \mathrm{D}_{0} \rightarrow{ }^{7} \mathrm{~F}_{2}$ transition and there is no significant change in the wavelength. This transition is induced by electric dipole transition and very sensitive to the historical preparation and environmental condition [13]. As shown in Fig. 1, the highest transition is the Eu complex blended with PVK, followed by PVK:PVDF-HfP and PVK:PVP. This can be best explained that the emission spectra produced by the samples are influenced by the environmental condition viz. type host polymer.

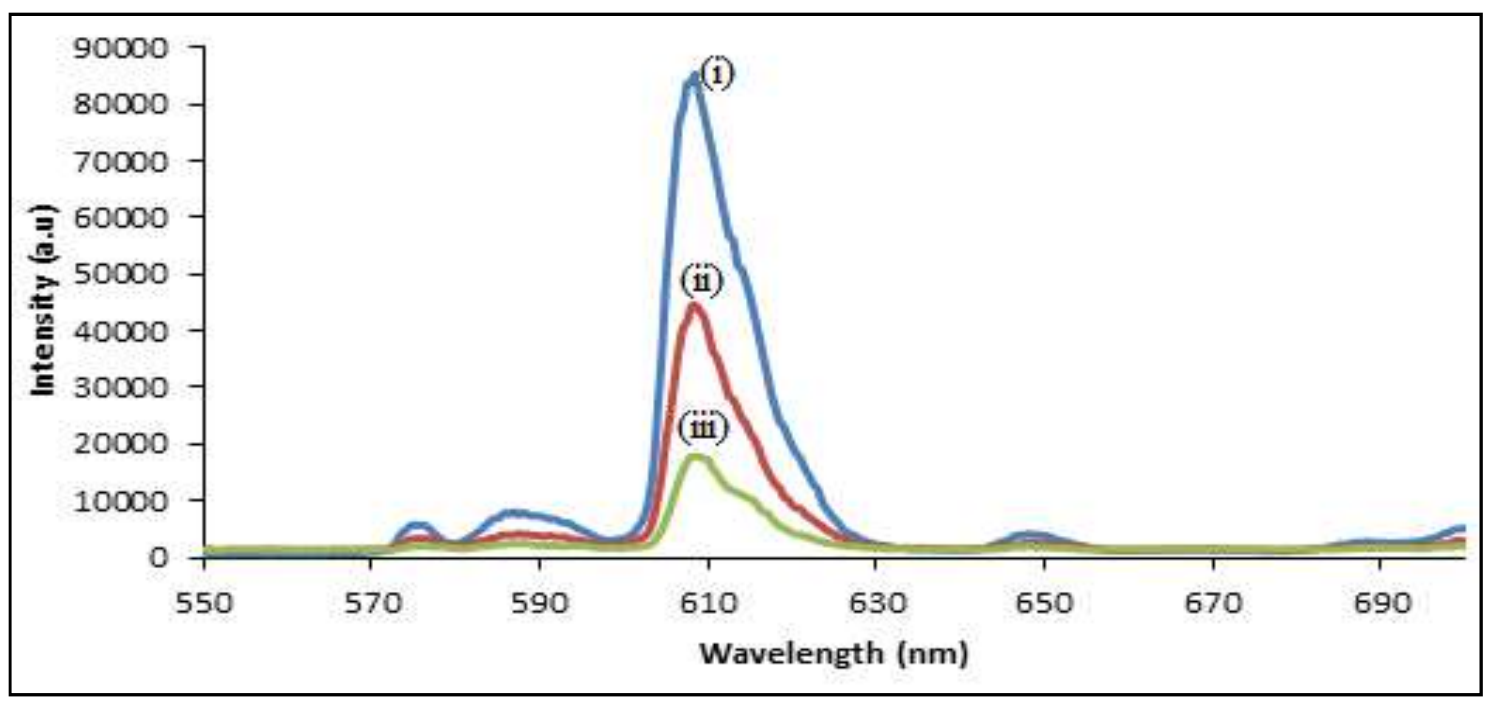

Fig.1.Emission spectra of (i) PVK, (ii) PVK:PVDF-HfP, and (iii) PVK:PVP blend with EuDBMPhen

The decay of ${ }^{5} \mathrm{D}_{0} \rightarrow^{7} \mathrm{~F}_{2}$ for Eu complex blended with PVK, PVK:PVDF-HfP and PVK: PVP was investigated by monitoring at the highest emission produced at $608.5 \mathrm{~nm}$. As shown in Fig. 2, the decay curve can be fitted using exponential decay function. The life time of Eu complex blended with PVK, PVK:PVDF-HfP and PVK: PVP is 0.353, 0.331 and $0.312 \mathrm{~ms}$ respectively. This life time for each samples is in the range of phosphorescence emission. The details of why different types of the host polymer gave different emission spectra and decay 
life time will be discussed in section 4 .

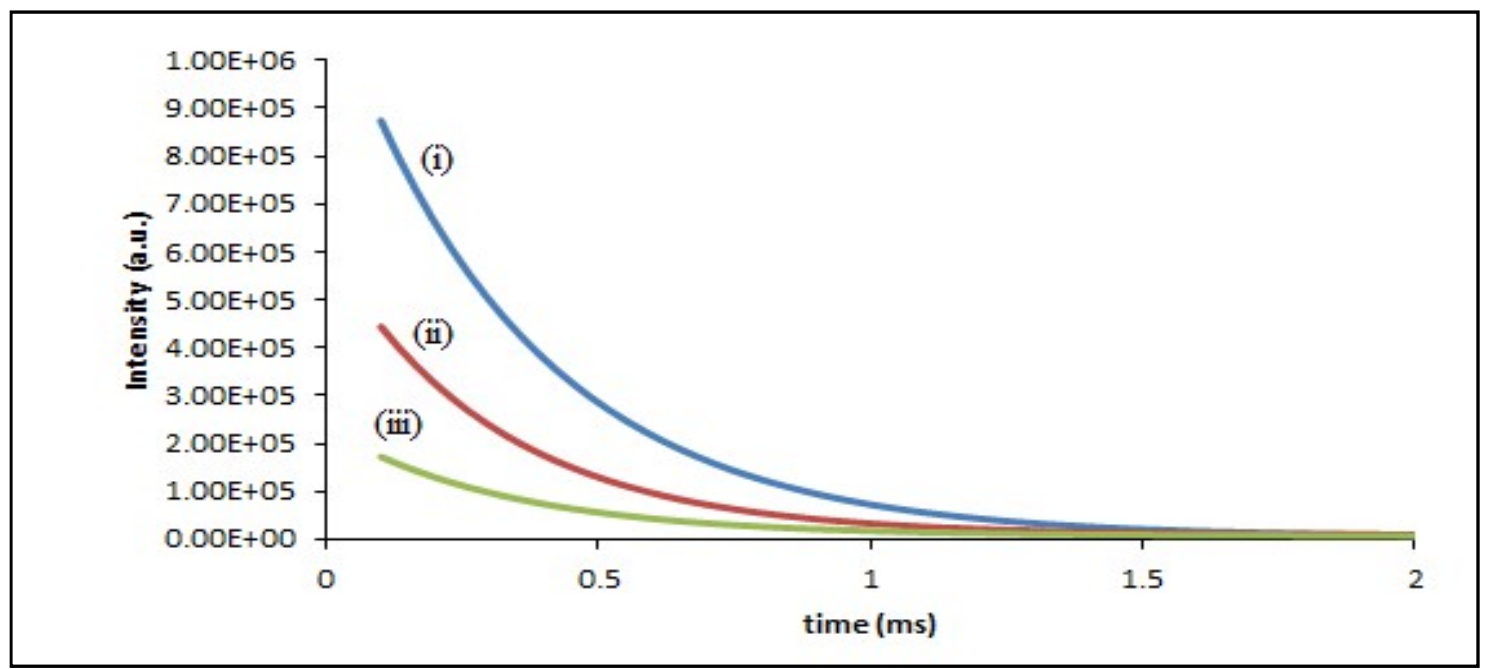

Fig.2. Emission spectra of (i) PVK, (ii) PVK:PVDF-HfP, and (iii) PVK:PVP blend with

\section{EuDBMPhen}

\section{SINGLET AND TRIPLET ANALYSIS OF PHOSPHORESCENCE EMISSION USING SEMI-EMPIRICAL CALCULATION}

Before we discuss the effects of phosphorescence emission in host polymers, it is better to investigate the energy transfer in Eu complex dye. EuDBMPhen is Europium complex consists of single 5-amino-1,10-phenanthroline (Phen) and three dibenzoylmethane (DBM) ligand group. Fig. 3 shows singlet-triplet energy levels of each Eu complex with Phen and $\mathrm{DBM}$ as a ligand or "antenna" for sensitization. The energy levels of $\mathrm{Eu}^{+3}$ are taken from the theoretical calculation in the literature [14]. When light is absorbed, the optical transition occurs at the ligand from ground state $S_{o}$ to first singlet excited state $S_{1}$. There are two probabilities in this case that the excited state will initially be induced either by Phen or DBM as shown in Fig. 3 (a) and (b) respectively. The intersystem crossing will be dropped from $\mathrm{S}_{1}$ to $T_{1}$ and hence the energy will be transferred from triplet state, $T_{1}$ via Phen or DBM to $\mathrm{Eu}^{+3} \mathrm{a}$ $4 f$ orbital. In [15] proposed that the energy difference $(\Delta \mathrm{E})$ between $\mathrm{T}_{1}$ ligand and $4 f$ orbital energy level for the effective energy transfer must be more than $0.245 \mathrm{eV}$ If the $\Delta \mathrm{E}$ is less than $0.245 \mathrm{eV}$, this energy has probability for thermally competitive back energy from $\mathrm{Eu}^{+3}$ to $\mathrm{T}_{1}$ ligand. While, based on [16] calculation the effective energy transfer between ligand and metal is about $\Delta \mathrm{E}>0.310 \mathrm{eV}$ According to [17], the triplet energy must be above $0.434 \mathrm{eV}$ 
from Lanthanide Ions. For Phen, $\Delta \mathrm{E}$ is less than $0.245 \mathrm{eV}$, while $\mathrm{DBM}$ has $\Delta \mathrm{E}$ more than $0.310 \mathrm{eV}$. Thus, it can be noted that the energy transfer process Phen to $\mathrm{Eu}^{3+}$ is less efficient compared with the DBM to $\mathrm{Eu}^{3+}$ as shown in Fig. 3 (a) and (b).

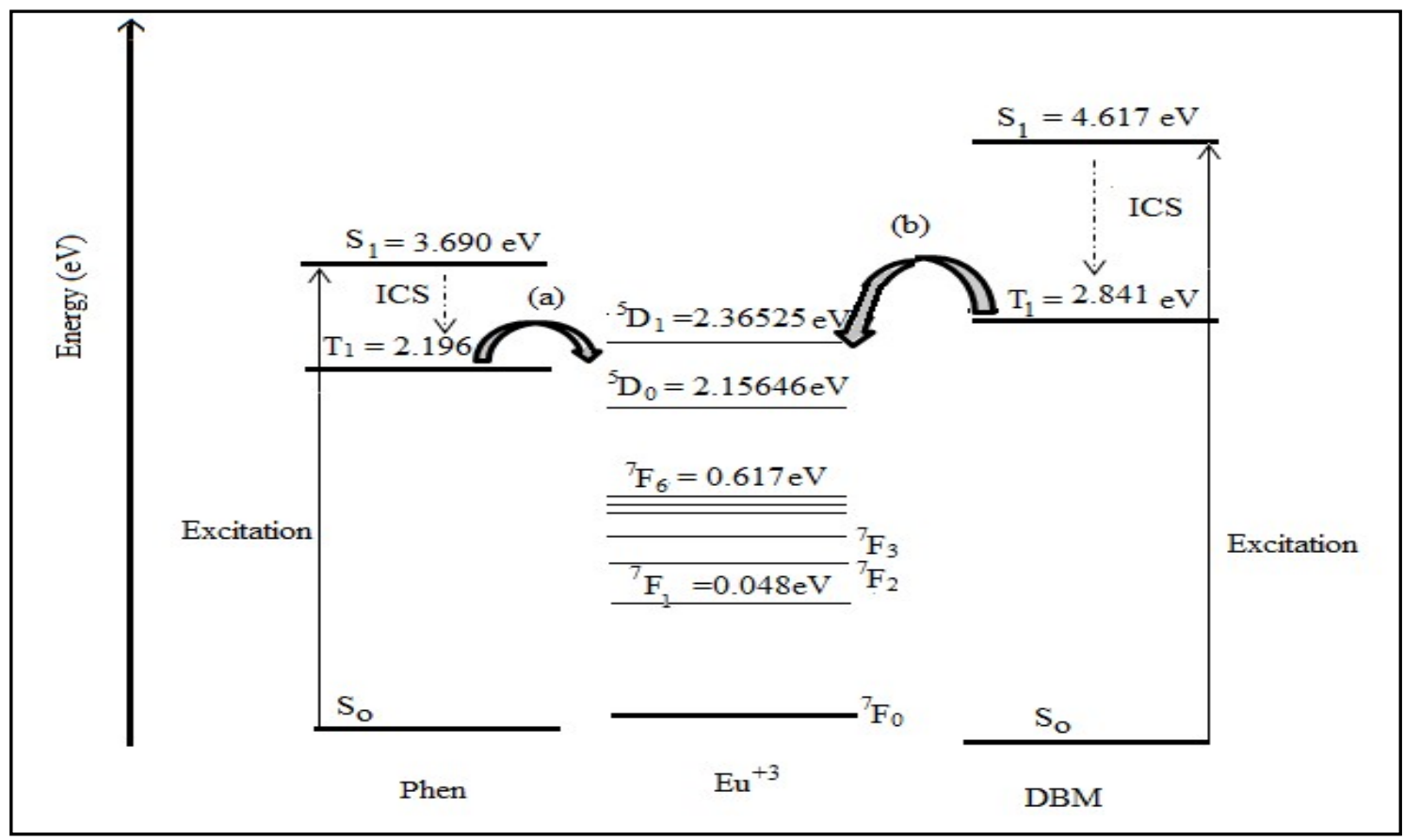

Fig.3. Singlet-Triplet energy levels of EuDBMPhen (a) energy transfer process from Phen to

$$
\mathrm{Eu}^{3+}(\mathrm{b}) \text { energy transfer process from } \mathrm{DBM} \text { to } \mathrm{Eu}^{3+}
$$

The host polymers such as PVK, PVK:PVDF-HfP and PVK:PVP act as donor which the host polymer providing an 'optical site sink' for the optical absorption process. Fig. 4 shows the electronic singlet-triplet energy level of PVK blending system with EuDBMPhen. Based on singlet-triplet energy levels, for PVK, the singlet energy level is lower compared to the DBM however is much higher compared to the Phen as shown in Fig. 3. This makes the probability to energy transfer effectively from host polymer to antenna Phen are much higher. The energy-level for describing phosphorescence emission in polymer blending system involves inter and intramolecule energy transfers. Based on Fig. 4, the energy levels is designated as follows, the ground state (level 1), the first singlet excited state of PVK (level 6), the singlet excited state of Phen (level 5), the triplet state of Phen (level 4), discrete ${ }^{5} \mathrm{D}_{0}$ Europium energy level (level 3) and discrete ${ }^{7} \mathrm{~F}_{2}$ Europium energy level (level 2). $\mathrm{A}_{61}, \mathrm{~A}_{51}$ and $\mathrm{A}_{41}$ is the radiationless transition probability for level $(6 \rightarrow 1),(5 \rightarrow 1)$ and $(4 \rightarrow 1)$ respectively. $\mathrm{S}_{65}$ is the intermolecule energy transfer from level 6 to level 5. $\mathrm{S}_{54}$ is the intramolecule singlet triplet 
(intersystem crossing system) transition. $\mathrm{S}_{43}$ is the energy transfer from triplet state of Phen to rare earth ions. $S_{32}$ and $S_{31}$ is transition probability for decay ${ }^{5} \mathrm{D}_{0}$ to ${ }^{7} \mathrm{~F}_{2}$ and ${ }^{5} \mathrm{D}_{0}$ to ground state respectively. $\mathrm{W}_{16}$ is the excitation transition probability for ground state to the highest excited state level 6.

$\mathrm{w}_{12}$ and $\mathrm{w}_{21}$ is the transition probability for thermal deactivation ion relaxation of ground state process. For the steady state condition, the steady state rate equation has been employed as follows

$\frac{d N_{6}}{d t}=W_{16} N_{1}-A_{61} N_{6}-S_{65} N_{6}=0$

$\frac{d N_{5}}{d t}=S_{65} N_{6}-A_{51} N_{5}-S_{54} N_{5}=0$

$\frac{d N_{4}}{d t}=S_{54} N_{5}-A_{41} N_{4}-S_{43} N_{4}=0$

$\frac{d N_{3}}{d t}=S_{43} N_{3}-S_{32} N_{3}-S_{31} N_{3}=0$

$\frac{d N_{2}}{d t}=S_{32} N_{3}+w_{12} N_{1}-w_{21} N_{2}=0$

(1e)

$N_{0}=N_{1}+N_{2}+N_{3}+N_{4}+N_{5}+N_{6}$

$\mathrm{N}_{\mathrm{j}}$ is the population of the $\mathrm{j}^{\text {th }}$ energy levels and $\mathrm{N}_{\mathrm{o}}$ is the total concentration of molecule. 


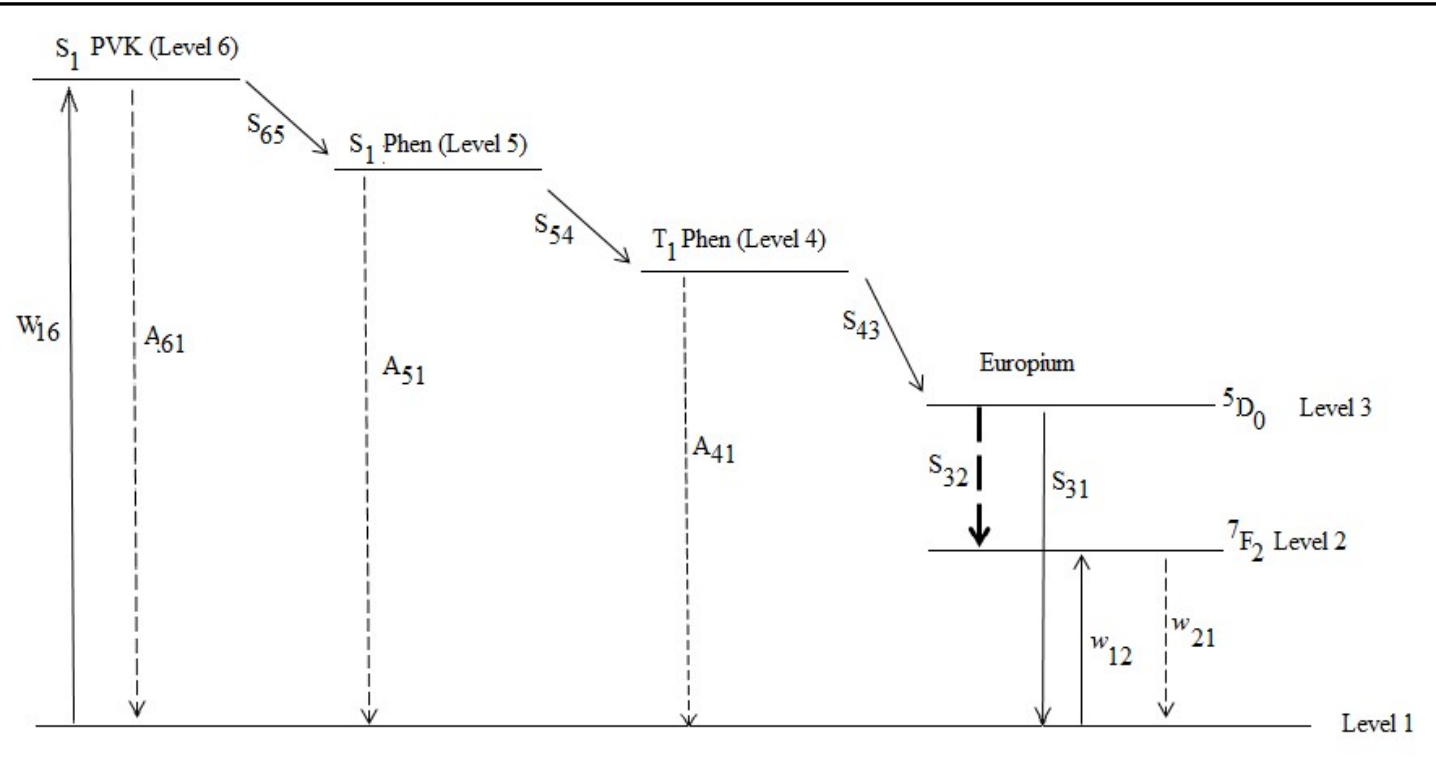

Fig.4. The electronic singlet-triplet energy level of PVK blending system with EuDBMPhen For the phosphorescence yield for the ${ }^{5} \mathrm{D}_{0} \rightarrow{ }^{7} \mathrm{~F}_{2}$ transition is defined as follows[18]

$\eta_{E_{u}}=S_{32} N_{3} / W_{16} N_{1}$

The solution of the Equation (2), the phosphorescence yield, $\eta_{\text {Eu }}$ become

$\eta_{E_{u}}=S_{32}\left(\frac{S_{43}}{S_{32}+S_{31}}\right)\left(\frac{S_{54}}{A_{41}+S_{43}}\right)\left(\frac{S_{65}}{A_{51}+S_{54}}\right) \frac{1}{A_{61}+S_{65}}$ (3)

From this calculation, phosphorescence yield for the ${ }^{5} \mathrm{D}_{0} \rightarrow{ }^{7} \mathrm{~F}_{2}$ transition is independent with excitation energy. In [21] also reported that the phosphorescence yield independent with excitation energy. Now, we consider two different cases, the first case that is the radiationless is dominant in this system

$\mathrm{A}_{41}>>\mathrm{S}_{43}, \mathrm{~A}_{41}>>\mathrm{S}_{43}, \mathrm{~A}_{51}>>\mathrm{S}_{54}$

The Equation (3) can be simplified as

$\eta_{E_{u}}=\left(\frac{S_{32}}{S_{32}+S_{31}}\right) \frac{S_{43} S_{54} S_{65}}{A_{41} A_{51} A_{61}}$

Based on the Equation (4), phosphorescence yield is reciprocal with the transition probability.

If the polymeric system encountersradiationless transition, then phosphorescence yield will be decreased according to numerical factor of transition probability. The radiationless transition might be due to phonon emission, conversion to the chemical energy, thermal deactivation or carriers trap [19]. 
Second case is the cascade energy transfer occurred in this system

$\mathrm{A}_{41}<<\mathrm{S}_{43}, \mathrm{~A}_{41}<<\mathrm{S}_{43}, \mathrm{~A}_{51}<<\mathrm{S}_{54}$

The Equation (3) can be simplified as

$$
\eta_{E_{u}}=\left(\frac{S_{32}}{S_{32}+S_{31}}\right)
$$

For this case, energy transfer are not involving radiationless transition. The energy will be transferred from high energy level to the next lowest energy level consecutively without energy lost. In this polymeric system, the phosphorescence yield is proportional with the transition probability for decay ${ }^{5} \mathrm{D}_{0}$ to ${ }^{7} \mathrm{~F}_{2}$.

PVDF-HfP and PVP has the highest $\mathrm{S}_{1}$ and $\mathrm{T}_{1}$ compared with DBM. For polymer blending system, another energy level of PVDF-HfP and PVP has been introduced which is labelled as level 7 as shown in Fig. 5. As same as before, for the steady state condition, the steady state rate equation has been employed as follows

$$
\begin{aligned}
& \frac{d N_{7}}{d t}=W_{17} N_{1}-A_{71} N_{6}-S_{76} N_{7}+A_{67} N_{6}=0 \\
& \frac{d N_{6}}{d t}=S_{76} N_{7}-\left(A_{61}+S_{65}+A_{67}\right) N_{6}=0
\end{aligned}
$$

$\frac{d N_{5}}{d t}=S_{65} N_{6}-A_{51} N_{5}-S_{54} N_{5}=0$

$$
\frac{d N_{4}}{d t}=S_{54} N_{5}-A_{41} N_{4}-S_{43} N_{4}=0
$$

$$
\frac{d N_{3}}{d t}=S_{43} N_{3}-S_{32} N_{3}-S_{31} N_{3}=0
$$

$$
\frac{d N_{2}}{d t}=S_{32} N_{3}+w_{12} N_{1}-w_{21} N_{2}=0
$$


$N_{0}=N_{1}+N_{2}+N_{3}+N_{4}+N_{5}+N_{6}+N_{7}$

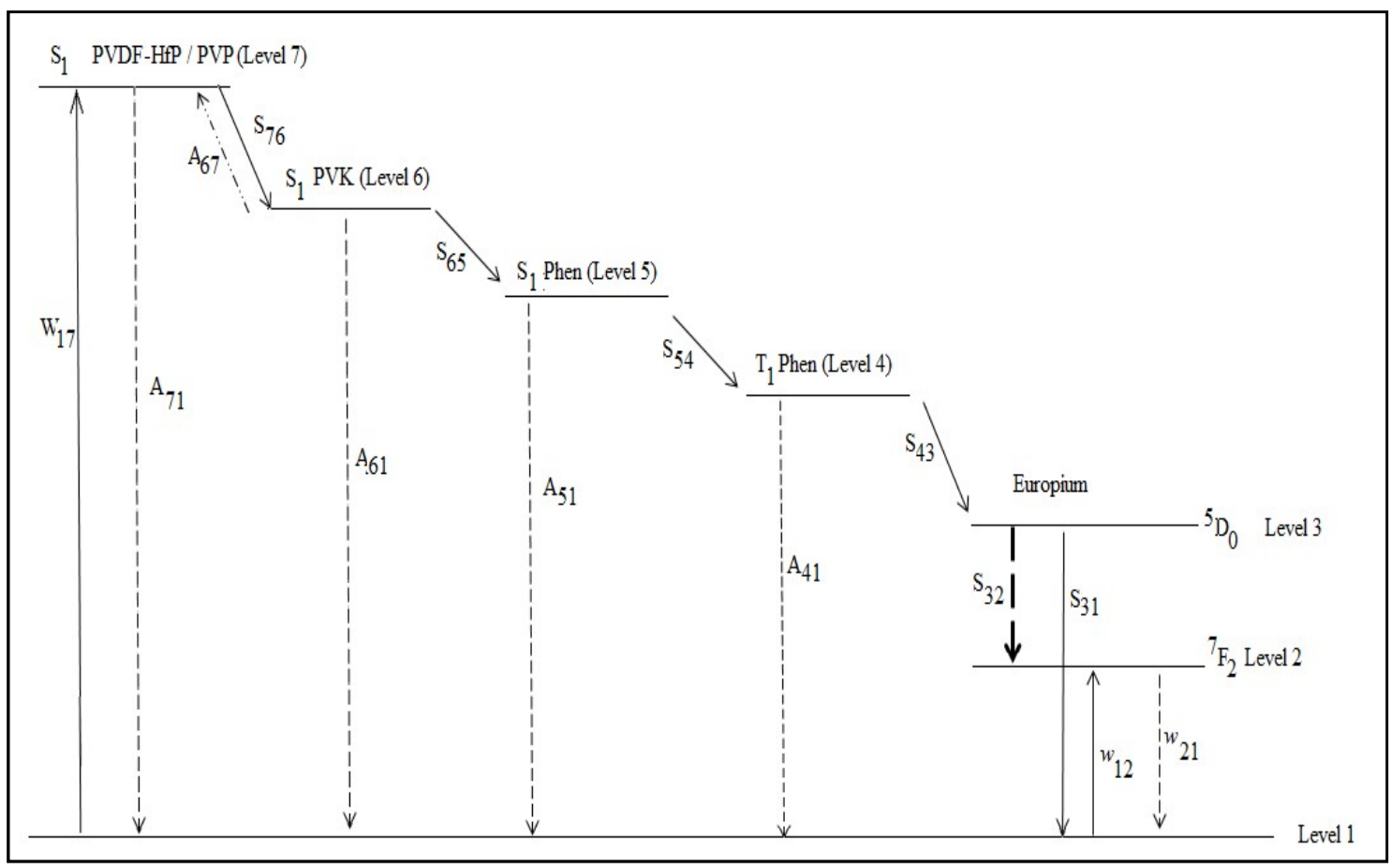

Fig.5. The electronic singlet-triplet energy level of PVDF-HfP/PVP blending system with

\section{EuDBMPhen}

The notation of Equation (6) is the same as equation 1 with a few modifications. $\mathrm{W}_{17}$ is the excitation transition probability for ground state to the highest excited state level $7 . \mathrm{S}_{76}$ and $\mathrm{A}_{67}$ is the reversible energy transfer for energy coupling[11]. $\mathrm{A}_{71}$ is the radiationless transition probability for level $(7 \rightarrow 1)$. In this system, the $\mathrm{N}_{\mathrm{j}}$ is the population involves seven energy levels. The phosphorescence yield becomes

$\eta_{E_{u}}=S_{32} \frac{S_{43} S_{54} S_{65}}{\left(S_{32}+S_{31}\right)\left(A_{41}+S_{43}\right)\left(A_{51}+S_{65}\right)}\left[\frac{S_{76}}{\left(A_{71}+S_{76}\right)\left(A_{61}+A_{67}+S_{65}\right)-\left(A_{67} S_{76}\right)}\right]$ (7)

Again, we consider two different cases. The first case is the radiationless that is dominant in this system

$\mathrm{A}_{41}>>\mathrm{S}_{43}, \mathrm{~A}_{41}>>\mathrm{S}_{43}, \mathrm{~A}_{51}>>\mathrm{S}_{65}, \mathrm{~A}_{71}>>\mathrm{S}_{76}$

Therefore, Equation (7) can be rewritten as

$\eta_{E_{u}}=\frac{S_{32}}{\left(S_{32}+S_{31}\right)}\left[\frac{S_{43} S_{54} S_{65} S_{76}}{A_{41} A_{51} A_{71}\left(A_{61}+A_{67}-1\right)}\right]$ (8)

Second case is the cascade energy transfer occurred in this system 
$\mathrm{A}_{41}<<\mathrm{S}_{43}, \mathrm{~A}_{41}<<\mathrm{S}_{43}, \mathrm{~A}_{51}<<\mathrm{S}_{54}$; the Equation (7) can be simplified as

$\eta_{E_{u}}=\frac{S_{32}}{\left(S_{32}+S_{31}\right)}\left[\frac{S_{65}}{S_{65}-1}\right]$

The experimental result, emission spectra in Fig. 1 despicts that EuDBMPhen blends with PVK:PVDF-HfP and PVK:PVP show lower amount of intensity compared with the EuDBMPhen blend. Based on the phosphorescence yield calculation for radiationless condition, the $\eta_{\mathrm{Eu}}$ of polymer blends have much radiationless transition probability occurred in polymer blending systems which will result in the value of $\eta_{\mathrm{Eu}}$ lower compared with the PVK. For a simple comparison in cascade energy transfer, the $\eta_{E u}$ for PVK blends with EuDBMPhen is directly depended on transition between the Eu levels. However, in polymer blending system, $\eta_{E u}$ has $\left[\mathrm{S}_{65} / \mathrm{S}_{65}-1\right]$ factor which lowering the phosphorescence yield. The plausible reason for the lowest amount of intensity inPVK:PVP blends with EuDBMPhen is the existence of $>\mathrm{C}=\mathrm{O}$ in $\mathrm{PVP}$ which oxygen creates the phosphorescence quenching site in complex Eu system [20].

\section{CONCLUSION}

The emission spectra of Europium complex blended with PVK, PVK:PVDF-HfP and PVK:PVP was investigated. The emission spectrum for all the samples showed the character of Eu complex. The highest transition was Eu complex which blended with PVK, followed by PVK:PVDF-HfP and PVK:PVP. This showed that the type of host polymer strongly depends upon the type of host polymer blends in Eu complex. Based on singlet-triplet energy levels, it has been calculated that there must be a competition in energy transfer or recombination carries to reduce the phosphorescence intensity in polymer blends.

\section{ACKNOWLEDGEMENTS}

This work was funded by 600-IRMI/FRGS 5/3 (88/2016) FRGS. The authors would like to thank Faculty of Applied Sciences, UiTM Perak and Ministry of Higher Education for supporting this project. 


\section{REFERENCES}

[1] Burroughes J H, Bradley D D, Brown A R, Marks R N, Mackay K, Friend R H, Burns P L, Holmes A B. Light-emitting diodes based on conjugated polymers. Nature, 1990, 347(6293):539-541

[2] Wang J, Peng J, Yao C, Lui R, Yao W, Zhang C, He M, Jiang C. Self-host blue-emitting iridium dendrimer for solution-processed non-doped phosphorescent organic light-emitting diodes with flat efficiency roll-off and less phase segregation. Organic Electronic, 2017, 45:49-56

[3] Kalyani N T, Swart H, Dhoble S J Review of literature on organic light-emitting diode devices. In N. T. Kalyani, H. Swart, \&S.J. Dhoble (Eds.), Principles and applications of organic light emitting diodes (OLEDs). Cambridge: Elsevier Ltd., 2017, pp. 171-203

[4] Marques L F, Santos H P, D'Oliveira K A, Botezine N A, Freitas M C R, Freire R O, Dutra J O L, Martins J S, Legnan C. New photo/electroluminescent Europium(III) $\beta$-diketonate complex containing a p,p'-disubstitutedbipyridine ligand: Synthesis, solid state characterization, theoretical and experimental spectroscopic studies. InorganicaChimicaActa, 2017, 458:28-438

[5] Shih P I, Shu C F, Tung Y L, Chi Y. Efficient white-light-emitting diodes based on poly (N-vinylcarbazole) doped with blue fluorescent and orange phosphorescent materials. Applied Physics Letters, 2006, 88(25):1-3

[6] Tao P, Li W, Zhang L J, Guo S, Zhao Q, Wang H, Wei B, Liu S, Zhou X, Yu Q, Xu B, Huan W. Facile synthesis of highly efficient lepidine-based phosphorescent iridium(III) complexes for yellow and white organic light-emitting diodes. Advanced Functional Materials, 2016, 26:881-894

[7] Alias A N, Kudin T T, Zabidi Z M, Harun M K, Yahya M Z. Optical characterization of luminescence polymer blends using Tauc/Davis-Mott model. Advanced Materials Research, 2012, 488:628-632

[8] Alias A N, Kudin T T, Zabidi Z M, Yahya M Z, Ali A M. Polarized absorption and dielectric spectra of poly (N-carbazole) blends. In IEEE Symposium on Business, Engineering and Industrial Applications, 2012, pp. 498-503

[9] Alias A N, Kudin T I, Zabidi Z M, Harun M K, Ali A M, Yahya M F. Optical studies of 
poly (N-carbazole)(PVK) blending with polyvinylpyrrolidone (PVP) using Tauc/Davis-Mott model. Advanced Materials Research, 2013, 652:527-531

[10] Alias A N, Kudin T I, Zabidi Z M, Harun M K, Ali A M, Yahya M F. Refractive index dispersion and optical dielectric properties of poly (N-carbazole)/poly (vinylpyrrolidone) blends. Advanced Materials Research, 2013, 652:532-536

[11] Alias A N, Zabidi Z M, Harun M K, Yahya M Z, Ali A M. Optical transition, excitation, and emission properties of poly (N-vinlycarbazole) blended with poly (vinylidene fluoride-co-hexafluoropropene) and polyvinylpyrrolidone. ActaPhysicaPolonica A, 2015, 127(4):1430-1433

[12] Alias A N, Ali A M M, Yahya M Z A. Electronic properties of polyvinlypyrrolidone(PVP) using semi-empirical calculation. In M. C. Wythers (Ed.), Advances in materials science research. New York: Nova Science, 2017, pp. 173-201

[13] Jiang L, Zheng J W, Chen W C, Ye J J, Mo L, Li Z Q, Hu L H, Zhang C H, Dai C Y. Tuning coordination environment: Better photophysical performance of europium(III) complex. Journal of Physical Chemistry C, 2017, 121(11):5925-5930

[14] Carnall W T, Fields P R, Rajnak K. Electronic energy levels of the trivalent lanthanide aquo ions. IV. Eu3+. Journal of Chemical Physics, 1968, 49(10):4450-4455

[15]Shi M, Li F, Yi T, Zhang D, Hu H, Huang C. Tuning the triplet energy levels of pyrazolone ligands to match the 5D0 level of europium (III). Inorganic Chemistry, 2005, 44(24):8929-8936

[16] Latva M, Takalo H, Mukkala V M, Matachescu C, Rodríguez-Ubis J C, Kankare J. Correlation between the lowest triplet state energy level of the ligand and lanthanide (III) luminescence quantum yield. Journal of Luminescence, 1997, 75(2):149-169

[17] Steemers F J, Verboom W, Reinhoudt D N, van der Tol E B, Verhoeven J W. New sensitizer-modified calix [4] arenes enabling near-UV excitation of complexed luminescent lanthanide ions. Journal of the American Chemical Society, 1995, 117(37):9408-9414

[18] Sato S, Wada M. Relations between intramolecular energy transfer efficiencies and triplet state energies in rare earth $\beta$-diketone chelates. Bulletin of the Chemical Society of Japan, 1970, 43(7):1955-1962

[19] Shimizu M, Kimura A, Sakaguchi H. Room-temperature phosphorescence of crystalline 
1,4-Bis(aroyl)-2,5-dibromobenzenes. European Journal of Organic Chemistry, 2016, $3: 467-473$

[20] Liu L, Zhang W, Li X, Wu X F, Yang C, Liu Y D, He L, Lu Y L, Xu R W, Zhang X J, Zhang L Q. Preparation and luminescence properties of Sm (TTA) 3 phen/NBR composites. Composites Science and Technology, 2007, 67(10):2199-2207

[21] Samelson H, Lempicki A, Brecher C. Laser phenomena in europium chelates. II. Kinetics and optical pumping in europium benzoylacetonate. Journal of Chemical Physics, 1964, 40(9):2553-2558

\section{How to cite this article:}

Alias A N, Zabidi Z M, Ali A M M, Yahya M Z A. Effect of host polymer blends to phosphorescence emission. J. Fundam. Appl. Sci., 2017, 9(6S), 524-537. 